\title{
Congenital or berry aneurysm in the optic nerve
}

\author{
RUSSELL V. MILLISER, STEPHEN R. GREENBERG, \\ AND BENJAMIN H. NEIMAN \\ From the Department of Pathology, The Chicago Medical School, University of Health \\ Sciences, Chicago, Illinois, The Department of Pathology, The MacNeal Memorial \\ Hospital, Berwyn, Illinois, and The Department of Pathology, The Cook County \\ Hospital, Chicago, Illinois, U.S.A.
}

SYNOPSIS A case is presented of a 67-year-old woman with hypertension and arteriosclerosis who died from cerebral infarction. There was an associated arteriosclerotic aneurysm of the right vertebral artery, and incidental congenital or berry aneurysms of the anterior communicating and left internal carotid or anterior cerebral artery. The latter aneurysm was situated within the left optic nerve.

Single or multiple small saccular aneurysms on the basal cerebral arteries are relatively common lesions. Gore, in the chapter on blood and lymphatic vessels in Anderson's 'Textbook of pathology' (1966) states that they occur with greater frequency than all other non-traumatic and non-mycotic aneurysms of extracranial arteries. Such aneurysms within the substance of the central nervous system are much less common. Most of these have a fairly definite inflammatory aetiology. A survey of the literature has revealed no case in which an aneurysm was situated within the substance of the optic nerve.

The subject of intracranial aneurysms has been extensively documented, but we felt that the unusual site of this lesion justified its being briefly reported. Because many recent publications include reviews of the literature on this subject, no attempt will be made here to do so. Stehbens (1963) in a study of 1,364 necropsies presents an excellent review with his interpretation of the pathogenetic factors involved in the formation of aneurysms of cerebral arteries.

\section{CASE REPORT}

A 67-year-old woman was admitted to the Cook County Hospital on 6 May 1966 with the chief complaint of frequent chest pains radiating to the left arm and a dry, unproductive cough. On the day of admission, she became unconscious while at home. Her history indicated a stroke three years before the present admission. She denied the use of alcohol or tobacco. A physical examin-

Received for publication 22 August 1967. ation contributed nothing. A neurological examination disclosed a comatose patient with facial weakness, eyes deviating to the right; the right pupil was dilated and sluggish to light. There was flaccid paralysis of the left upper and lower extremities with positive ankle clonus and the Babinski sign on the left side. She died on 10 May 1967.

\section{NECROPSY FINDINGS}

Pertinent abnormalities included hypertensive hypertrophy of the left ventricle with focal myocardial fibrosis and moderate chronic cor pulmonale. The brain showed a fairly typical hypertensive encephalopathy with diffuse atrophy, gliosis, and vascular sclerosis. There was an extensive infarction in the right cerebral hemisphere. The basal vessels showed advanced atherosclerosis with a fusiform aneurysm of the right vertebral artery.

There was also a small saccular (congenital berry) aneurysm on the anterior communicating artery (Fig. 1).

Of considerable incidental interest was the smooth, firm, fusiform enlargement measuring $9 \times 9 \times 8 \mathrm{~mm}$ at the left optic nerve $1 \mathrm{~cm}$ anterior to the chiasma. The sella turcica and the pituitary gland were not involved by the lesion.

The neoplasm which was suspected from gross inspection was not supported by histopathological examination. Instead a small saccular aneurysm was found extending into the optic nerve. The vascular lesion was almost completely covered by the nerve (Fig. 2). Since this was unsuspected at the time of necropsy, the artery from which the aneurysm arose was not determined. Its situation between the 


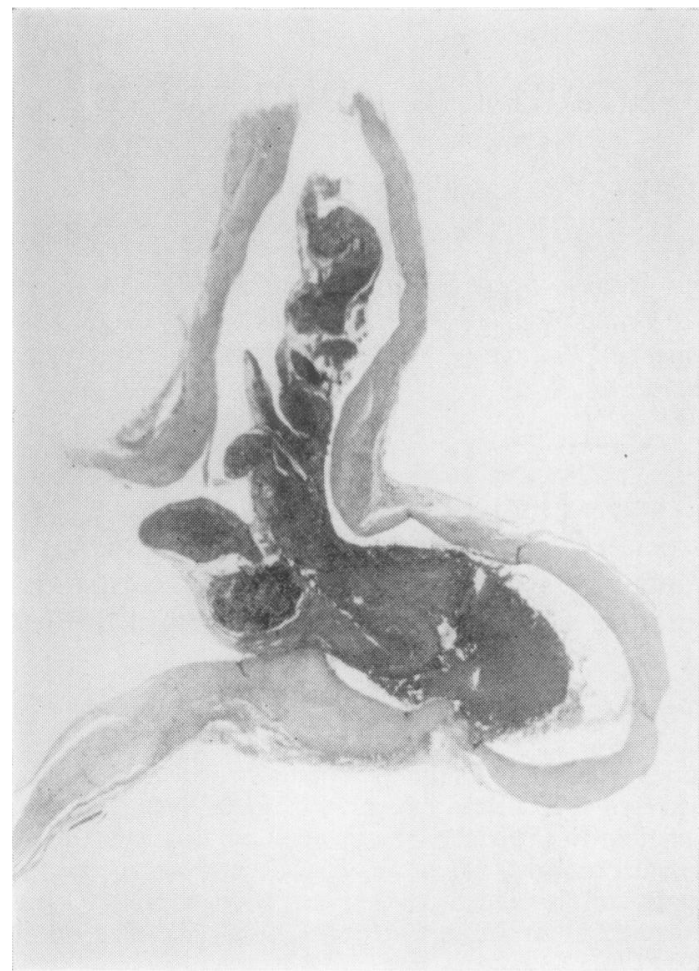

FIG. 1.

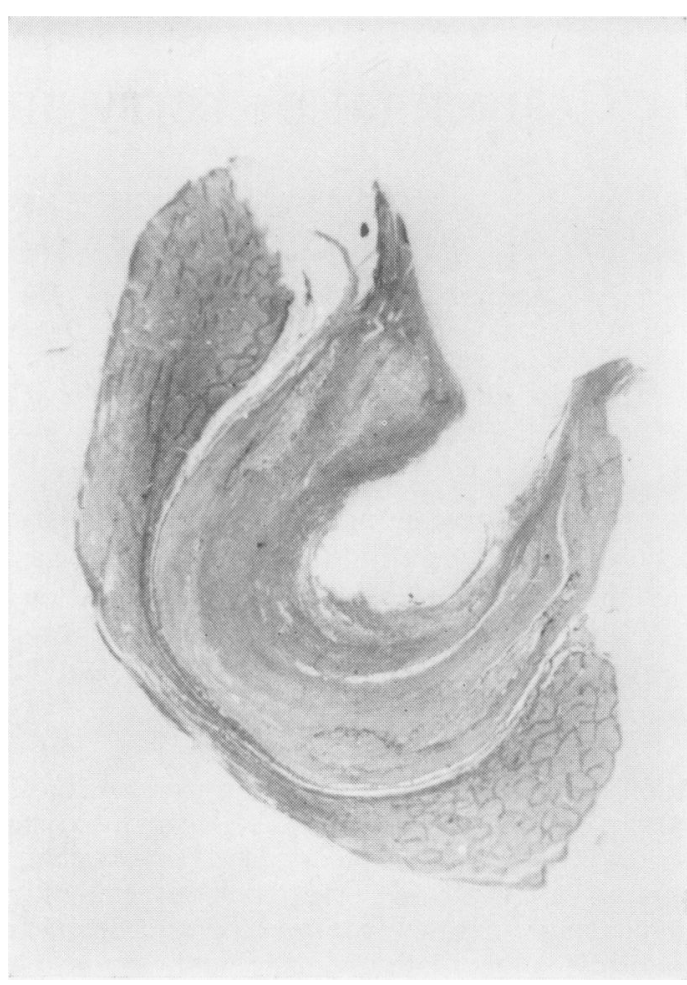

FIG. 2.

FIG. 1. A low-power view of the aneurysm of the anterior communicating artery. Haematoxylin and eosin $\times 10$. FIG. 2. A low-power view of the aneurysm of the anterior cerebral and/or internal carotid artery. Haematoxylin and $\operatorname{eosin} \times 10$.

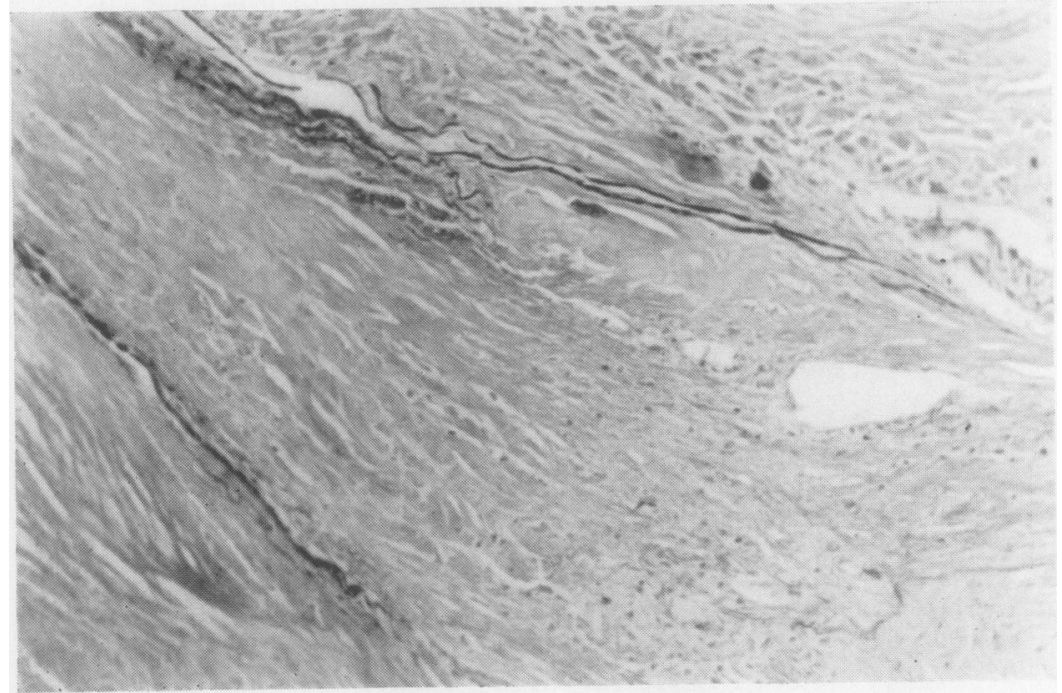

FIG. 3. The fragmentation 0 of the elastic fibres in the neck of the aneurysm. 응 Van Gieson-Weigert elastic tissue stain $\times 100$. 100. $\frac{7}{0}$

FIG. 3 . 
chiasma and the emergence of the nerve from the orbit suggested that the aneurysm arose from the anterior cerebral, at or near its origin from the internal carotid, or the internal carotid itself. Only a very small amount of the parent vessel was available for study.

The thickened intima of the parent artery was composed of collagenous and myxomatous connective tissue. It showed numerous empty spaces some of which were slit-like and were assumed to have contained lipids and/or cholesterol. There were a few large lipophages present. The intima was greatly thickened at its junction with the sac and in the sac proper. In the sac, the structure was similar, but the collagen bundles were more irregularly arranged with extensive hyalinization, and the myxomatous component and lipophages more abundant.

Near the luminal surface at the blind end of the sac were found several endothelial-lined spaces, containing blood cells, suggesting organization of an old thrombus. Further supporting this concept was the presence of a more recent thrombus partially filling the lumen of the sac. The outer portions of the intima showed several small areas of dystrophic calcification.

The internal elastic membrane of the parent artery, at the origin of the sac, showed an apparent increase of elastic fibres, and was extensively split and fragmented (Fig. 3). It decreased in amount in the neck of the sac and disappeared completely in the sac proper. The media of the parent vessel showed a great decrease of smooth muscle and apparently an increase of collagenous fibres. There was no suggestion of an external elastic membrane. No smooth muscle was seen in the wall of the sac proper. There was no increase of smooth muscle with a blunted, rounded ending at the junction with the sac as is sometimes seen in such aneurysms. The adventitia of the parent artery was abundant and composed of rather dense collagenous tissue. Vasa vasorum were present. The adventitia was thickened at the junction with the sac but around the sac proper was much thinner, more densely collagenous and blended imperceptibly with the meninges of the optic nerve. Small vessels were present but it was impossible to determine whether these were vasa vasorum or small vessels in the pia arachnoid of the nerve. Some of these vessels had thickened walls while others were thinwalled and appeared compressed and flattened.

The aneurysm extended into the optic nerve compressing it into a C-shaped structure which almost completely covered the aneurysm (Fig. 2). The thinnest portion of the nerve as seen in cross section was directly opposite the fundus of the sac where it was less than half the thickness of those portions near the neck of the aneurysm. The pia arachnoid seemed more densely fibrous than normal and blended with the adventitia of the aneurysm. The interfasicular stroma of the nerve was increased in amount, except at its thinnest portion, where it seemed decreased. The small blood vessels in this stroma were flattened and decreased in number in the thinnest and most compressed portion. The normal fascicles of the nerve were greatly thinned and compressed in the region of greatest pressure. With ordinary stains (haematoxylin and eosin and Van Gieson) both the size and the number of nerve fibres seemed decreased in the more distorted and compressed fascicles. The number of cells (assumed to be glia cells) between the nerve fibres and within the fascicles were less numerous in the more compressed fascicles. A few psammomma bodies were present but these were not more numerous in the more compressed portions of the nerve. Silver and myelin sheath stains of the nerve confirmed the above findings.

\section{COMMENT}

The pathogenesis of aneurysms has been extensively reviewed by Walker and Allegre (1954) and Stehbens (1963) and is always associated with pathology in the vessel wall. These changes in the wall may be inflammatory, retrogressive (degenerative), congenital, proliferative (compensatory) or some combination of these. The inflammatory group can be divided roughly into a granulomatous type, in which the vessel wall is generally involved from without inwards, and the embolic and/or septicaemic type, generally due to pyogenic organisms, where the involvement is from within outwards. Rupture of the latter group is frequently early when the aneurysms are still small. There was no clinical or pathological evidence to support such a pathogenesis in our case.

Atherosclerosis is the outstanding factor in the regressive or degenerative group and our case did have hypertension and moderately severe arteriosclerosis. Our study strongly suggests that the vertebral artery aneurysm belongs to this category. However, it is not possible to eliminate completely a preexisting congenital defect in the wall of this vessel. The fusiform shape of this aneurysm grossly is more characteristic of the arteriosclerotic type than the congenital. Walker and Allegre (1954) point out that the significance of arteriosclerotic changes as causative factors of intracranial aneurysms are underestimated by many investigators. The anterior communicating and 'optic nerve' aneurysms appear to be congenital in origin. Their saccular 
shape is compatible with this concept. The absence of significant systemic inflammation, including syphilis, practically eliminates a granulomatous or mycotic origin. The retrogressive or degenerative changes seen resemble arteriosclerosis and may be of causative significance, but we believe them to be secondary, and probably of lesser importance.
We wish to express our appreciation to Dr Paul Szanto, Director of Pathology at Cook County Hospital, 을 for making this case available.

REFERENCES

Gore, I. (1966). In Pathology, edited by W. A. D. Anderson, 5th ed., vol. 1, p. 570. Mosby, St Louis Kimpton, London.

Stehbens, W. E. (1963). Arch. Path., 75, 45.
Walker, A. E., and Allegre, G. W. (1954). J. Neuropath. exp. Neurol., 13, 248. 